\title{
The Growth of Single Crystals of InGaSe Compounds, Their X-Ray-Phase Analysis, Electronic Structure and Optical Functions
}

\author{
Eldar Gojaev, Zakir Jahangirli, Pyuruza Alieva, Khatira Khalilova, Tahir Musaev \\ Azerbaijan Technical University, Baku, Azerbaijan \\ Email: geldar-04@mail.ru
}

Received July 16, 2012; revised August 20, 2012; accepted September 24, 2012

\begin{abstract}
Growing of InGaSe $\mathrm{S}_{2}$ single crystals has been carried out by employing the Brijman-Stockbargor methods. On the base of X-ray-analysis it has been found that the given phase is crystallized into tetragonal symmetry. The temperature dependence of electric conductivity has been studied. Band structure has been computed. Optical functions, effective masses of electrons and holes have been calculated. Origin of formation of valence and conductance bands as well as the width of forbidden band for $\mathrm{InGaSe}_{2}$ have been determined.
\end{abstract}

Keywords: Band Structure; Optical Functions; $\mathrm{InGaSe}_{2}$

\section{Introduction}

During last years great quantity of works has been devoted to the study of $A^{111} B^{V 1}$ type semiconductor compounds that is related with interest to their specific physical properties. A nature of chemical bonds in these compounds causes specific features as layered and chain structure which are important from both fundamental and technological points of view. In particular, layered semiconductors create rising interest with their possible optoelectronic application [1]. Chain compounds of this type possess higher coefficients of photo- and tensilesensitivity as well as the switching properties with memory [2-5]. Their two-dimensional structural anisotropy as well as the specific optical and photoelectric properties attract researchers working for successfully learning of the processes occurred in these compounds.

The $A^{111} B^{111} C_{2}^{V 1}$ type chalkogenides are representatives of layered and chain $A^{111} B^{V 1}$ type semiconductors. Although, in latest years the ternary semiconductor chalkogenides are intensively studied, however a negligible number of papers have been devoted to the study of $\mathrm{InGaSe}_{2}$ and $\mathrm{InGaSe}_{2}$ [6-10]. Similarly to other semiconductor compounds these materials may have large range of application broadening from the use in solar elements up to nonlinear optical systems. In addition, they may easily intercalated by impurity ions, atoms and molecules assuming real predictions for development of the monitored super-lattices on their basis.

First X-ray structural researches showed the $\mathrm{InGaSe}_{2}$ like other representatives of $A^{111} B^{111} C_{2}^{V 1}$ type compounds possess tetragonal structure [11]. In more latest researches these crystals were considered to be in monoclinic symmetry [10], at the same time information was given about multi-type modifications with other crystalline structures. In this connection a structure of single crystal of $\mathrm{InGaSe}_{2}$ has been investigated in detail, the HRTEM electron diffraction of selected region (SAED) has been carried out. In these investigations was used the HF-200 TEM equipped with emitting-field gun. To obtain the images of back scattering of electrons which is observed upon chemical contrast between phases with different chemical compositions the Cambridge S-100 SEM was used [10].

The present paper is devoted to the growth of single crystals of InGaSe ${ }_{2}$ compounds, their X-ray-phaseanalysis, investigation the electro-conductivity, computation the band structure and optical functions.

\section{The Experimental Method}

The single crystals of $\mathrm{InGaSe}_{2}$ have been undergone to growth by the Brijemena-Staockbarger method. The double-wall quartz ampoules with evacuated external part were used for minimization of the convection effects. First, ampoules were cleaned by use the mixture of fluoric acid and distilled water (with volume proportion 1:3) and then with pure alcohol. After performance of the chemical purification the evacuated ampoules were put into dryer at $1000^{\circ} \mathrm{C}$ for 12 hours. After dehumidification 
under $1000^{\circ} \mathrm{C}$ temperature the ampoules were cooled and filled with highly cleaned elements in stechiometric proportions.

To reduce the risk of explosion of ampoule the blend was slowly heated (with speed of $0.5^{\circ}$ minute) from temperatures $200^{\circ} \mathrm{C}-250^{\circ} \mathrm{C}$. The melt was kept under $950^{\circ} \mathrm{C}$ temperature during 48 hours for obtaining homogeneous. Then the furnace was undergone to cooling with speed of $0.6 \mathrm{~mm} /$ hour with replacement from hot place to colder one with $350^{\circ} \mathrm{C}$.

After obtaining of the single crystals they were undergone to the X-ray-phase analysis after their obtaining. Decoding of diffractogram of $\mathrm{InGaSe}_{2}$ showed that the given phase is crystallized into tetragonal symmetry with lattice parameters $a=8.0138 \AA$ and $c=6.953 \AA$. Diffractograms are shown in Figure 1, results of X-rayphase analysis are given in Table $\mathbf{1}$.

For analysis of the electric properties the samples of single crystals were obtained from large pieces (sizes of $2.2 \mathrm{~mm} \times 1.0 \mathrm{~mm} \times 1.0 \mathrm{~mm}$ ) by cutting with diamond knife. The process of polishing with rotary disk was nec-

Table 1. Results of X-ray-phase analysis of $\operatorname{InGaSe}_{2}$.

\begin{tabular}{ccccc}
\hline$\theta$ & $d_{e x}(\AA)$ & $d_{\text {cal }}(\AA)$ & $h k l$ & $J / J_{0}$ \\
\hline $12^{\circ} 56^{\prime}$ & 4.0015 & 4.0019 & 200 & 6 \\
$12^{\circ} 2^{\prime}$ & 3.1750 & 3.1801 & 211 & 100 \\
$15^{\circ} 48^{\prime}$ & 2.8321 & 2.8317 & 220 & 13 \\
$17^{\circ} 4^{\prime}$ & 2.6364 & 2.6275 & 202 & 17 \\
$17^{\circ} 42^{\prime}$ & 2.5338 & 2.5360 & 310 & 14 \\
$24^{\circ} 21^{\prime}$ & 1.8679 & 1.8724 & 411 & 40 \\
$27^{\circ} 39^{\prime}$ & 1.6614 & 1.6592 & 332 & 36 \\
$34^{\circ} 7^{\prime}$ & 1.3785 & 1.3746 & 530 & 8 \\
$49^{\circ} 53^{\prime}$ & 1.1780 & 1.1780 & 532 & 10 \\
\hline
\end{tabular}

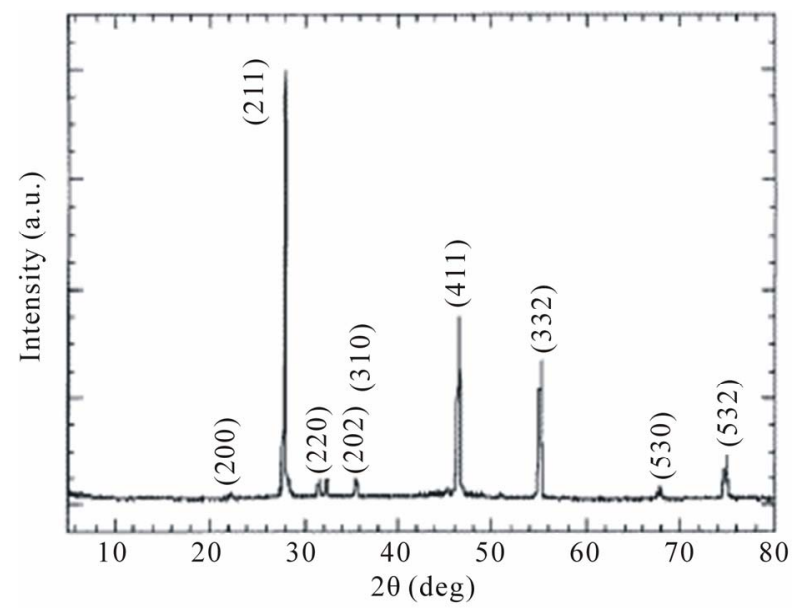

Figure 1. Diffractogram of $\mathrm{InGaSe}_{2}$ single crystal. essary for obtaining the bulk samples with parallel surfaces. To obtain the best polishing process the mixture of $1 \mathrm{~mm} \alpha$-aluminum and $0.005 \mathrm{~mm} \beta$-aluminum were used. After polishing the samples were washed in pure ethyl alcohol then twice in distilled hot water. Then the samples were put for dehumidification without any thermal processing. Accurate determination of the sizes of sample that is needed for exact calculation of the electric properties has been carried out by optical microscope.

\section{Results and Discussions}

By use the thermoelectric energetic graduation we observed that the investigated samples demonstrate the $\mathrm{p}$ type conductivity. Temperature dependence of conductivity was measured in the $300-900 \mathrm{~K}$ temperature range. Note that, up to present paper the studies of temperature dependence for electric resistivity of $\mathrm{InGaSe}_{2}$ single crystal have been carried out in the temperature range of 4.2 $300 \mathrm{~K}$. The results indicate exponential character of dependence in agreement with relationship $\exp (-E / k T)$ where $k$ is the Boltzmann constant, $E$ refers to the activation energy. Thermal energy of activation for majority carriers (holes) is $51.8 \mathrm{meV}$. The resistivity of $\rho=1.51 \times 10^{-3} \Omega \cdot \mathrm{cm}$ was measured at a room temperature.

In the present paper the temperature dependence of electric conductivity of $\mathrm{InGaSe}_{2}$ has been investigated in the temperature range of $300-900 \mathrm{~K}$. From results presented in Figure 2 it follows that in the investigated range of temperature the electric conductivity increases with temperature. Width of forbidden gap determined from the high-temperature slope of temperature dependence for this phase is in good agreement with that one calculated on the basis of band structure of $\mathrm{InGaSe}_{2}$.

Width of forbidden gap determined from the high-temperature slope of temperature dependence for this phase is in good agreement with that one calculated on the basis of band structure of $\mathrm{InGaSe}_{2}$. In this paper the band spectrum has been studied, the genezis of electron states was determined and optical functions have been calculated for $\mathrm{InGaSe}_{2}$ crystal. We have carried out calculation of the electron states on the basis of theory of functional of local electron density. For this aim the method of pseudo-potential was employed on the basis of plane waves. Nonlocalized pseudo-potentials were constructed according to the diagram presented in [12]. Screening of ion pseudo-potential was made through the function given by Khabbard and Shem [13]. In expansion of wave function of electrons 3800 plane waves have been used. Calculation was made at symmetrical points $\Gamma, T, N, P$ as well as over the lines connecting them.

The lattice's parameters are determined through minimization the ionic energy and the structural parameters are optimized by use the Gelman-Feynmann forces. The 


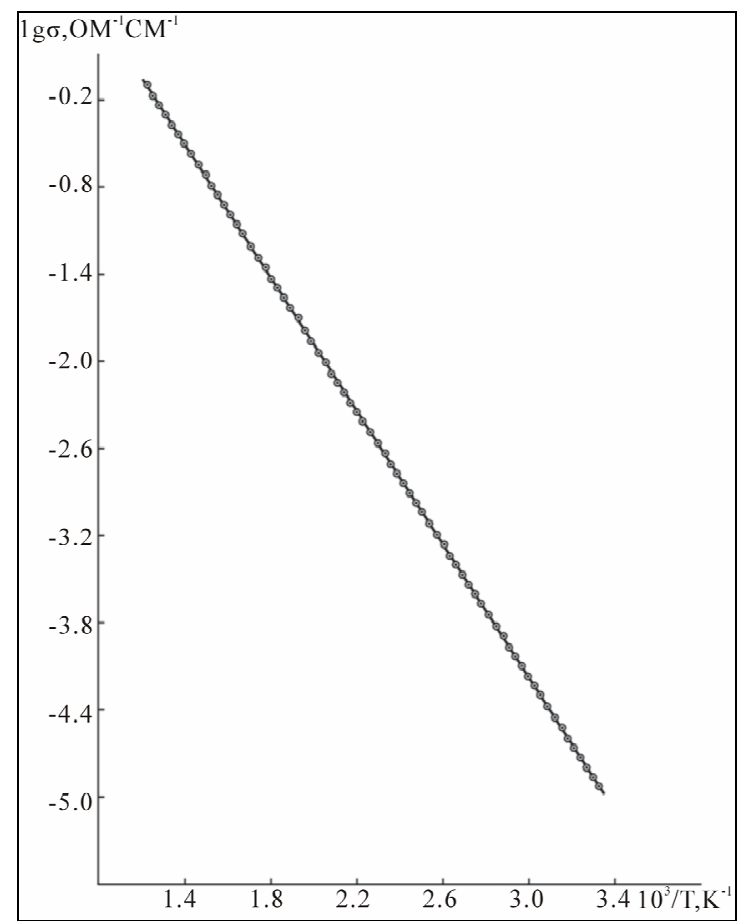

Figure 2. Temperature dependence of electric conductivity for InGaSe 2 single crystal.

minimization process was occurred providing condition

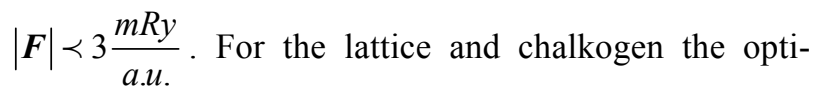
mized parameters are $a=8.0138 \AA, c=6.953 \AA$ and $x=$ 0.1720 respectively.

The table of unreduced and binary unreduced representations of wave vector groups for space group $D_{4 h}^{18}$ were obtained in [14]. Here also are demonstrated condition of agreement between unreduced representations of simple groups and double-value group $D_{4 h}^{18}$ and view of Brillouin zone for volume-centered tetragonal lattice. The band spectrum of $\mathrm{InGaSe}_{2}$ is presented in Figure 3. Where the top of valence band was taken as the ground energy. The main feature of valence zone is that it consists of three pronounced sub-bands separated by forbidden values of energy. The lower sub-band consisting of four zones is about $-10 /(-11) \mathrm{eV}$ and apart from others by wide energy gap of order $-6 \mathrm{eV}$. Results of theoretical-group analysis carried out by use of the symmetry properties of $\mathrm{InGaSe}_{2}$ crystal show that these valence bands are obliged with their origin to $5 \mathrm{~s}$ states of Se. Next group located in energy level near $-5 \mathrm{eV}$ consists of four valence bands originated from s-states of In and $\mathrm{Ga}$ atoms. The top subzone consisting of ten bands of $\approx 5 \mathrm{eV}$ width is originated from p-states of In, Ga and Se atoms.

In [10] the X-ray photoemission spectrum of valence band of $\mathrm{InGaSe}_{2}$ is demonstrated. Experimentally was shown that photoemission spectrum consists of three more pronounced regions. The observed peaks about

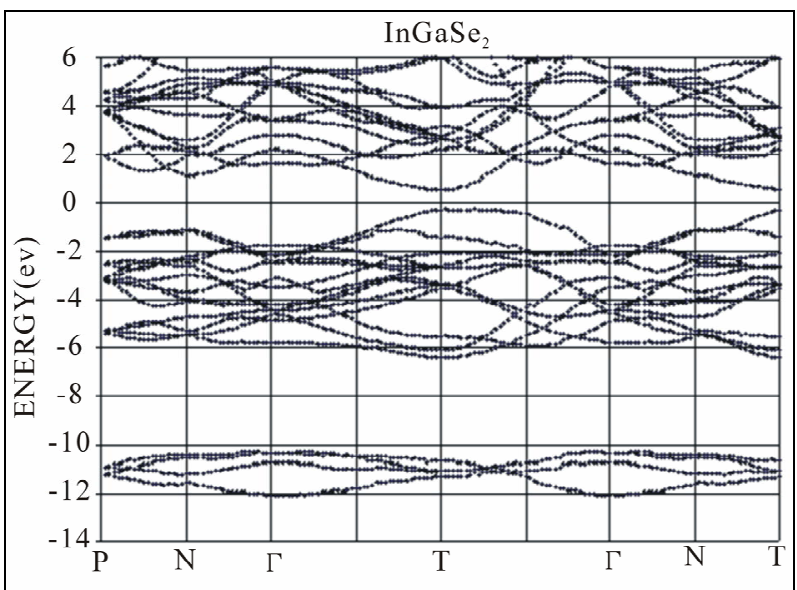

Figure 3. Band structure of InGaSe 2 compound.

$-13.5 \mathrm{eV}$ and $-4 \mathrm{eV}$ correspond to s-states of Se and GaSe bonds respectively. Complex of features about $-5 / 0$ $\mathrm{eV}$ corresponds to both $\mathrm{Ga}-\mathrm{Ga}$ and $\mathrm{Ga}-\mathrm{Se}$ bonds that is in a good agreement with our band spectrum calculations and theoretical-group analysis.

Top of valence band as well as the bottom of conductivity band are located at point $T$ with high symmetry on the surface of Brillouin zone and lower energetic transition occurs at this point. Other minimum of conductivity band is located at high symmetrical point $N$. For the width of forbidden band our calculations gives $0.95 \mathrm{eV}$ and extremity of fundamental absorption is originated by direct transitions.

Optical functions for $\mathrm{InGaSe}_{2}$ were calculated by known method given in [15]. For determination the spectral dependence of imaginary part of complex dielectric constant we have used relationship

$$
\varepsilon_{i}(E)=\frac{N}{E^{2}} \sum_{k \in B Z} \sum_{v_{i} c}\left|\boldsymbol{e} \cdot \boldsymbol{M}_{c v}(\boldsymbol{k})\right|^{2} \delta\left(E_{c}(\boldsymbol{k})-E_{v}(\boldsymbol{k})-E\right)(1)
$$

here integral

$$
\boldsymbol{e} \cdot \boldsymbol{M}_{c v}(\boldsymbol{k})=\boldsymbol{e} \cdot \int \psi_{c k}^{*}(\boldsymbol{r})(-\mathrm{i} \hbar \nabla) \psi_{v k}(\boldsymbol{r}) \mathrm{d}^{3} r .
$$

is taken over the volume of elementary cell of crystal and indicates matrix element of operator of linear momentum $p=-\mathrm{i} \hbar \nabla$; indices $v$ and $c$ numerate the states of valence and conductivity bands respectively; $e$ indicates unit vector of polarization. During summation over the Brillouin zone the elementary cell of inverse lattice was divided into 64 parts of equal volumes and $k$-points were randomly selected in them. Totally were taken 3000 points and those gave continuously (regular) histogram. Constant $N$ is determined from condition of normalization of histogram

$$
\int_{0}^{\infty} E \varepsilon_{i}(E) \mathrm{d} E=\frac{\pi}{2 \hbar^{2}} \omega_{p}^{2}=\frac{\pi}{2 \hbar^{2}} \cdot \frac{4 \pi n_{e} e^{2}}{m_{e}}
$$


where $\omega_{p}$ refers to plasma frequency of electrons and $n_{e}$ indicates mean density of electrons in crystal.

Histogram was plotted with step of $0.2 \mathrm{eV}$ and covered all transitions between zones $v \rightarrow c$ with energy up to $12 \mathrm{eV}$. Starting from small structure in histogram about $12 \mathrm{eV}$ dependence $\varepsilon_{i}(E)$ was extrapolated according to formula $\varepsilon_{i}(E)_{E \rightarrow \infty} \approx E^{-3} \mid$. Transitions from most lower bands having Se-5s origin as can be seen partially are found within the range of investigated spectral region. These bands are located far and as our studies show, broadening of spectral region and thus involves high energetic transitions from these bands into upper states of conductivity band does not give to dielectric constant significant contribution distinguished from extrapolation. Real part of dielectric constant was determined from Kramer-Kronig integral dispersion relation

$$
\varepsilon_{r}(E)=1+\frac{2}{\pi} P \int_{0}^{\infty} E^{\prime} \varepsilon_{i}\left(E^{\prime}\right) \cdot \frac{\mathrm{d} E^{\prime}}{E^{\prime 2}-E^{2}}
$$

where $P$ indicates the integral of principal meaning. Results of spectral functions calculations of $\mathrm{InGaSe}_{2}$ compounds are given for $e / / c$ and $e \perp c$ polarizations in Figures 4 and 5 respectively. As can be seen from Figure 4 calculated curves rapidly rise up to maximum. According to these figures maximum in the $\varepsilon_{r}(E)$ spectrum upon polarizations.

However in the $\varepsilon_{i}(E)$ spectrum for both polarizations maximum of main fringe which is related to transitions from upper part of valence zone to downer part of conductivity zone corresponds to $3.5 \mathrm{eV}$ energy. For $e \perp c$ type polarization in the $\varepsilon_{i}(E)$ four additional peaks of $4.25 \mathrm{eV}, 5.55 \mathrm{eV}, 5.95 \mathrm{eV}$ and $6.45 \mathrm{eV}$ energies have been found. In the case of e//c type polarization additional peaks are weak and located at energies $4.05 \mathrm{eV}$, $5.08 \mathrm{eV}$ and $5.75 \mathrm{eV}$.

Maximum value of $\varepsilon_{i}$ upon $e / / c$ and $e \perp c$ polarizations are 24.9 and 14.95 respectively and such a difference belongs to chain $\mathrm{InGaSe}_{2}$ crystals with high ani-

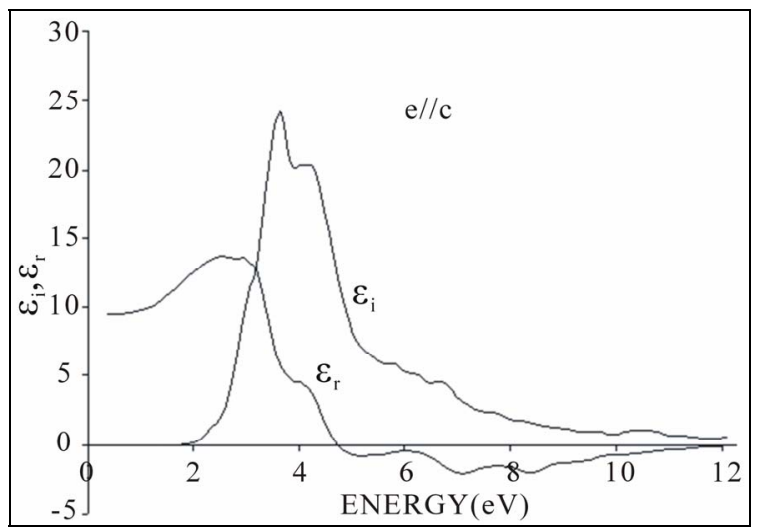

Figure 4. Spectral dependence of imaginary $\left(\varepsilon_{i}\right)$ and real $\left(\varepsilon_{r}\right)$ parts of dielectric constant of $\mathrm{InGaSe}_{2}$ compound in the case of $e / / c$ polarization.

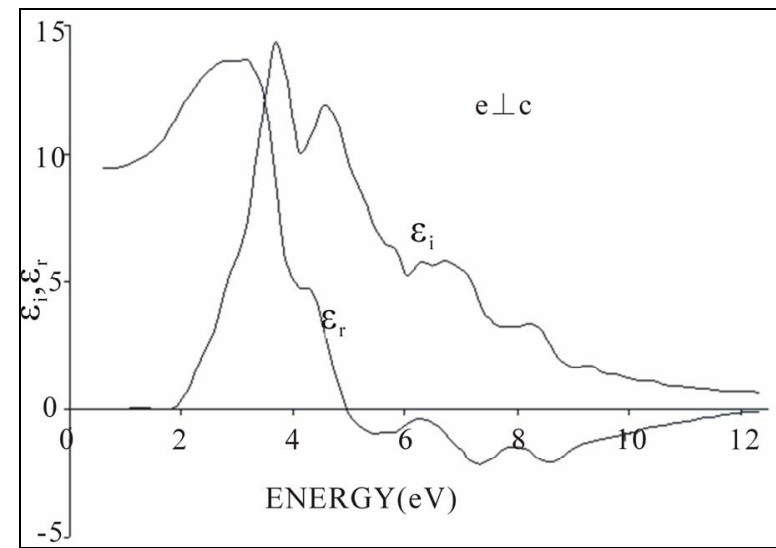

Figure 5. Spectral dependence of imaginary $\left(\varepsilon_{i}\right)$ and real $\left(\varepsilon_{r}\right)$ parts of dielectric constant of InGaSe $_{2}$ compound in the case of $e \perp c$ polarization.

sotropy. Limit values of $\varepsilon_{r}(E=0)$ are obtained as 9.5 and 9.1 upon $e / / c$ and $e \perp c$ type polarizations respectively.

Components of tensor of inverse effective mass were determined by the formula $m_{0}$ is the resting mass of electron; $\delta_{i j}$ refers to Kroneker symbols; $\left\langle n_{1} k_{0}\left|P_{i}\right| n_{1}^{\prime} k_{0}\right\rangle$ is the matrix element of operator of linear momentum $P_{i}=-\mathrm{i} \hbar \frac{\partial}{\partial x_{i}}$ at the extreme point $k_{0}$ for $n$ and $n^{\prime}$ electron zones; $\left.\left.\| n_{1} k_{0}\right\rangle\right\rangle$ is the wave function of electron

$$
\left\langle n_{1} k_{0}\left|P_{i}\right| n_{1}^{\prime} k_{0}\right\rangle \frac{1}{\lambda} \int_{\lambda} \varphi_{n k_{0}}^{*}(r) P_{i} \phi_{n^{\prime} k_{0}}(r) \mathrm{d}^{3} r
$$

$\lambda$ refers to volume of elementary cell Energetic spectrum and $E_{n}\left(k_{0}\right)$ and appropriate wave function $\varphi_{n k_{0}}(r)$ at extreme point $k_{0}$ is determined from the Schrodinger single electronic equation

In the basis of plane waves

$$
\begin{aligned}
& \sum_{G^{\prime}}\left[\frac{\hbar\left(k_{0}+G^{\prime}\right)}{2 m_{e}} \delta_{G G^{\prime}}+V\left(k_{0}+G_{1} k_{0}+G^{\prime}\right)\right] \varphi_{n}\left(k_{0}+G^{\prime}\right) \\
& =E_{n k_{0}} \varphi_{n}\left(k_{0}+G\right)-V\left(k_{0}+G_{1} k_{0}+G^{\prime}\right)
\end{aligned}
$$

$v(k)$ is the Fourier view of crystalline pseudo-potential.

Calculation of $\mathrm{InGaSe}_{2}$ zone structure shows that both maximum of valence and minimum of conductivity zones are located at point $\mathrm{T}$ of high symmetry

$k_{0}=0.5 b_{1}-0.5 b_{2}+0.5 b_{3}$. Here $b_{1}, b_{2}, b_{3}$ are basis translations of inverse lattice.

In our calculations components of tensor of inverse effective mass of electron were measured with an accuracy to $0.01 m_{0}$.

$$
\left(\frac{m_{0}}{m_{e}^{*}}\right)=\left[\begin{array}{ccc}
2.32 & 0 & 0 \\
0 & 2.32 & 0 \\
0 & 0 & 2.95
\end{array}\right]
$$


Tensor components of inverse effective mass of holes is given by

$$
\left(\frac{m_{0}}{m_{n}^{*}}\right)=\left[\begin{array}{ccc}
2.23 & 0 & 0 \\
0 & 2.23 & 0 \\
0 & 0 & 0.32
\end{array}\right]
$$

As can be seen tensors of inverse effective mass of both electrons and holes have diagonal view and therefore isoenergetic surfaces are described by rotational ellipsoids that is correspond to symmetry of $\mathrm{InGaSe}_{2}$ crystal. Please do not revise any of the current designations.

\section{Conclusion}

Growing of single crystals, their X-ray-phase analysis, investigation of the temperature dependence of electric conductivity and determination of the width of $\mathrm{InGaSe}_{2}$ forbidden band have been carried out. The electron structure was computed in the range of theory of functional of local electron density by employing the method of pseudopotential on the base of plane waves. Origin of valence and conductivity bands and width of forbidden band for $\mathrm{InGaSe}_{2}$ have been determined. Real and imaginary parts of dielectric constant were calculated for the polarizations $e / / c$ and $e \perp c$ in the energy range of $(0-12) \mathrm{eV}$. Also effective masses of electrons and holes for $\mathrm{InGaSe}_{2}$ were calculated.

\section{REFERENCES}

[1] V. Capozzi, L. Pavesi and J. L. Staehli, "Exiton-Carrier Scattering in Gallium Selenide," Physical Review, Vol. 47, No. 11, 1993, pp. 6340-6349.

[2] G. B. Abdullaev, V. D. Rustamov, M. Z. Ismailov, K. M. Bunnayev and S. M. Bidzinova, "The Way of Regulation of the Sensitivity of Strain Gauges," Patent AS539215 (USSR), 1976.

[3] A. G. B. bdullaev, A. Q. Abdullaev, G. D. Guseinov, Z. A. Asimov and A. M. Karnaukhov, "Semiconductor Switches," Patent AS 607502 (USSR), 1978.

[4] G. B. Abdullaev, G. D. Guseinov, M. K. Aliev, I. V. Alekseev, E. M. K. Boorman and V. L. Photoresistor, "Semiconductor," Patent AS 272986 (USSR), 1970.

[5] M. Hanias and A. N. Anagnostopoulos, "Negative-Differential-Resistance Effects in the $\mathrm{TlGaTe}_{2}$ Ternary Semi-
Conductor," Physical Review B, Vol. 160, No. 8, 1993, pp. 4261-4266.

[6] E. M. Gojayev, G. S. Orujov and D. M. Kafarova, "Band Structure and Dielectric Permittivity of $\mathrm{TlGaTe}_{2} \mathrm{Com}-$ pound," Solid State Physics, Vol. 46, No. 5, 2004, pp. 811813.

[7] G. Orudzhev, N. Mamedov, H. Uchici, N. Yamamoto, S. Iida, E. Gojaev, H. Toyota and F. Hashimzade, "Band Structure and Optical Function of Ternary Chain TlInSe, Journal of Physics and Chemistry of Solids, Vol. 64, No. 9-10, 2003, p. 1703.

[8] E. M. Gojayev, G. S. Orujov, R. A. Kerimova and E. A. Allahyarov, "Band Structure and Optical Properties of a Chain Compound TlInTe, ${ }_{2}$, Solid State Physics, Vol. 48, No. 1, 2006, pp. 40-43.

[9] M. Mobarak, H. Berger, G. F. Lorusso, V. Capozzi, G. Perna, M. M. Ibrahim and G. Margaritondo, "The Growth and Properties of Single Crystals of $\mathrm{GaInTe}_{2}$, a Ternary Chalkogenide Semiconductor," Journal of Physics D: Applied Physics, Vol. 31, No. 12, 1998, pp. 1433-1437.

[10] M. Mobarak, H. Berger, G. F. Lorusso, V. Capozzi, G. Perna, M. M. Ibrahim and G. Margaritondo, "The Growth and Characterization of $\mathrm{GaInSe}_{2}$ Single Crystals," Journal of Physics D: Applied Physics, Vol. 30, No. 18, 1997, pp. 2509-2516. doi:10.1088/0022-3727/30/18/003

[11] D. Müller, G. Eulenberger and H. Hahn, "Uber Tirnare Thallium-Chaluogenide Mittalliumselenide Structures," Journal of Inorganic and General Chemistry, Vol. 398, No. 2, 1997, pp. 207-220.

[12] V. Heine, M. Cohen and D. Weir, "The Pseudopotential Theory," Mir, Moscow, 1973.

[13] G. B. Bachelet, D. R. Haman and V. Shlüter, "Psevdopotensials That Work: From $\mathrm{H}$ to Pu," Physical Review B, Vol. 26, No. 8, 1982, pp. 4199-4228.

[14] F. M. Hashimzade, "The Symmetry of Energy Bands in TlSe Type Crystals," Physics, Vol. 12, No. 12, 1960, pp. 3040-3044.

[15] X. Gonze, J.-M. Beuken, R. Caracas, F. Detraux, M. Fuchs, G.-M. Rignanese, L. Sindic, M. Verstraete, G. Zerah, F. Jollet, M. Torrent, A. Roy, M. Mikami, J.-Y. Ghosez and D. C. Raty, "First-Principles Computation of Material Properties: The ABINIT Software Project," Computational Materials Science, Vol. 25, No. 3, 2002, pp. 478-492. doi:10.1016/S0927-0256(02)00325-7 